(61.7\% vs $24.9 \%)$, myopathy $(21.3 \%$ vs $10.2 \%)$ and pulmonary hypertension $(34.8 \%$ vs $22.3 \%)$. Significantly more SSc ILD patients were treated with cyclophosphamide, mycophenolate mofetil and azathioprine. Survival Kaplan-Meier curve patiets demonstrate reduced survival in patients with ILD $(p<0.01)$. Five years survival rates between years 2000 and 2015 have not changed significant. Mortality risk assessed with Cox regression analysis in the whole group was significantly higher in males, Arabs, DcSSc, elder age, heart and muscle involvement, and treatment with CYC. In the ILD group, the mortality was significantly higher in Arabs (3.3 times), elder age (8.9 times), presence of PAH (3.1 times) and treatment with CYC (2.8 times) compared to patients without ILD.

Conclusion: In our SSc cohort, ILD affected about third of patients and had major impact on patients' outcome. Male gender, Arab nationality, elder age, DcSSc, topoisomerase antibodies, heart and muscle involvement were significantly associated with worst prognosis. Despite active approach to assessing and treatment, survival rates of patients with SSc and SSc-ILD have not improved in last decades. Enrichment of the cohort with severe patients to a tertiary center due to reference bias and low efficacy of existed immunomodulatory drugs in SSc and in SSc related ILD particularly, could explain our results.

Disclosure of Interests: None declared

DOI: 10.1136/annrheumdis-2021-eular.3105

\section{POS0872 CLINICAL FEATURES AND OUTCOME OF 1054 PATIENTS WITH SYSTEMIC SCLEROSIS: AN ANALYSIS OF THE PORTUGUESE REUMA.PT REGISTRY FOR SCLERODERMA (REUMA.PT/SSC)}

R. Freitas ${ }^{1}$, P. Martins ${ }^{2}$, E. Dourado ${ }^{2}$, M. J. Salvador ${ }^{3,4}$, T. Santiago $^{3,4}$, I. Cordeiro' ${ }^{2}$, B. M. Fernandes ${ }^{5}$, F. Guimarães ${ }^{6}$, S. Garcia ${ }^{5}$, B. Samões ${ }^{7}$, N. Gonçalves ${ }^{8}$, M. H. Fernandes Lourenco ${ }^{8}$, A. S. Pinto ${ }^{9}$, M. Rocha ${ }^{10}$,

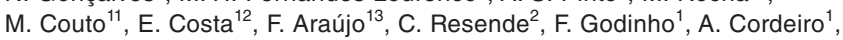
M. J. Santos ${ }^{1,14} .^{1}$ Hospital Garcia de Orta, Rheumatology Department, Almada, Portugal; ${ }^{2}$ Centro Hospitalar Lisboa Norte, Rheumatology Department, Lisboa, Portugal; ${ }^{3}$ Centro Hospitalar e Universitário de Coimbra, Rheumatology Department, Coimbra, Portugal; ${ }^{4}$ University of Coimbra, Rheumatology Department, Coimbra, Portugal; ${ }^{5}$ São João Universitary Hospital Center, Rheumatology Department, Porto, Portugal; ${ }^{6}$ Centro Hospitalar do Alto Minho, Rheumatology Department, Ponte de Lima, Portugal; ${ }^{7}$ Centro Hospitalar Vila Nova de Gaia, Rheumatology Department, Vila Nova de Gaia, Portugal; ${ }^{8}$ Centro Hospitalar Lisboa Ocidental, Rheumatology Department, Lisboa, Portugal; ${ }^{9}$ Unidade de Saúde Local da Guarda, Rheumatology Department, Guarda, Portugal; ${ }^{10}$ Centro Hospitalar Universitário do Algarve, Rheumatology Department, Faro, Portugal; ${ }^{11}$ Centro Hospitalar Tondela - Viseu, Rheumatology Department, Viseu, Portugal; ${ }^{12}$ Hospital de Braga, Rheumatology Department, Braga, Portugal; ${ }^{13}$ Hospital de Sant'Ana, Rheumatology Department, Parede, Portugal; ${ }^{14}$ Lisbon University, Medical School, Lisboa, Portugal

Background: Systemic sclerosis (SSc) may present distinctive manifestations and survival in different ethnic and geographic groups.

Objectives: To describe the clinical features, treatments, and survival of adult SSc patients registered in Reuma.pt/SSc.

Methods: Demographic features, SSc subsets, fulfilment of classification criteria, clinical and immunologic characteristics, comorbidities, medication and deaths were reviewed. Survival was calculated for patients included in the registry within the first 2 years of diagnosis.

Results: In total, 1054 patients were included, $87.5 \%$ female, mean age at diagnosis $52.7 \pm 14.8$ years. The most common subset was limited cutaneous (Ic)SSc (56.3\%), followed by diffuse cutaneous (dc)SSc (17.5\%), preclinical SSc $(13 \%)$, overlap syndrome $(9.8 \%)$ and SSc sine scleroderma $(3.3 \%)$. Raynaud's phenomenon $(93.4 \%)$ and skin thickening $(76.9 \%)$ were the most observed manifestations. Gastrointestinal $(62.8 \%$ vs $47.8 \%)$, pulmonary $(59.5 \%$ vs $23 \%)$ and cardiac (12.8\% vs $6.9 \%)$ involvement were significantly more prevalent in dcSSc compared to IcSSc (Table 1). $52.5 \%$ of patients were ACA positive and $21 \%$ anti-topoisomerase positive, with significant differences between IcSSc and dcSSc. One third of patients was treated with immunomodulators, $53.6 \%$ with vasodilators, $23 \%$ received glucocorticoids and $2.3 \%$ biologics.

During the median follow-up 12.4 years, 83 deaths $(7.9 \%)$ were verified. The overall 1,2 and 5 years survival was $98.0 \%, 96.8 \%$ and $92.6 \%$ respectively, without significant differences between IcSSc and dcSSc (Figure 1).

Conclusion: Reuma.pt/SSc register is useful in routine patient monitoring and contributes to improve knowledge about this rare and complex disease. Clinical features of Portuguese SSc patients are similar to what has been described in other populations although the overall 5 -year survival in recently diagnosed patients appears to be higher than previously reported.
Table 1. Cumulative clinical and immunologic characteristics of Portuguese SSc patients

\begin{tabular}{|c|c|c|c|c|}
\hline & & $\begin{array}{l}\text { Limited cuta- } \\
\text { neous SSc }\end{array}$ & $\begin{array}{l}\text { Diffuse cuta- } \\
\text { neous SSc }\end{array}$ & \\
\hline Clinical and immunologic features & $\mathrm{N}=1054$ & & & \\
\hline Skin involvement $-\mathrm{N}(\%) \mathrm{N}=987$ & \multicolumn{2}{|c|}{688 (90.6)525 (90.7) } & $180(100)$ & $<0.01$ \\
\hline Skin thickening * $-N(\%) N=962$ & \multicolumn{2}{|c|}{680 (76.9)512 (88.9) } & $180(100)$ & $<0.01$ \\
\hline Digital ulcers $-\mathrm{N}(\%) \mathrm{N}=970$ & \multicolumn{2}{|c|}{$325(33.5) 186(34.7)$} & $4(51.5)$ & $<0.01$ \\
\hline Raynaud's Phenomenon - N (\%) N=1010 & \multirow{2}{*}{\multicolumn{2}{|c|}{$\begin{array}{l}943(93.4) 539(95.7) \\
346(45.6) 247(42.7)\end{array}$}} & $157(92.4)$ & 0.06 \\
\hline Musculoskeletal involvement - N(\%) & & & $99(55)$ & $<0.01$ \\
\hline \multicolumn{5}{|l|}{$\mathrm{N}=972$} \\
\hline Cardiac involvement $-\mathrm{N}(\%)-\mathrm{N}=924$ & $71(7.7)$ & $36(6.9)$ & $19(12.8)$ & 0.02 \\
\hline Renal involvement $-\mathrm{N}(\%)-\mathrm{N}=917$ & $17(1.9)$ & $8 ! 1.5)$ & $6(4.1)$ & 0.07 \\
\hline \multicolumn{3}{|c|}{ Gastrointestinal involvement - N(\%) N=933508 (48.2)277 (47.8) } & $113(62.8)$ & $<0.01$ \\
\hline Pulmonary involvement - N(\%) N=915 & \multicolumn{2}{|c|}{$261(28.5) 119(23)$} & $88(59.5)$ & $<0.01$ \\
\hline $\mathrm{PAH}-\mathrm{N}(\%) \mathrm{N}=871$ & $14(1.6)$ & $10(2)$ & $1(0.7)$ & 0.23 \\
\hline Intersticial lung disease - N(\%) N=765 & \multicolumn{2}{|c|}{$218(28.5) 100(22.7)$} & $75(57.7)$ & $<0.01$ \\
\hline Antinuclear antibodies - $\mathrm{N}(\%) \mathrm{N}=1040$ & \multirow{2}{*}{\multicolumn{2}{|c|}{$\begin{array}{l}934(89.8) 522(90.2) \\
540(52.6) 383(67.1)\end{array}$}} & $154(88.5)$ & 0.57 \\
\hline Anti-centromere - N(\%) N= 1027 & & & $16(9.5)$ & $<0.01$ \\
\hline Anti-Scl70 - N(\%) N=1020 & $214(21)$ & $12(3.3)$ & $104(60.1)$ & $<0.01$ \\
\hline $\begin{array}{l}\text { Anti-RNA polymerase III - N(\%) N=710 } \\
\text { Comorbidities }\end{array}$ & $25(3.5)$ & $12(3.3)$ & $7(5.6)$ & 0.38 \\
\hline Hypertension - N(\%) N=431 & $117(27.1)$ & $76(29.7)$ & $67(20.7)$ & 0.1 \\
\hline Hyperlipidemia $-\mathrm{N}(\%) \mathrm{N}=431$ & $71(13.4)$ & $72(12.2)$ & $24(15.9)$ & 0.08 \\
\hline Neoplasia - N(\%) N=1054 & $29(2.8)$ & $12(2.1)$ & $7(3.9)$ & 0.14 \\
\hline
\end{tabular}

PDE-5 (phosdiasterase-5); PPIs (proton pump inhibitors); PAH-Pulmonary arterial hypertension confirmed by right heart catheterization. Immunomodulators includes Metothrexate Leflunomide, Hydroxycloroquine; Azathioprine, Mycophenolate Mofetil and Cyclophosphamide; ${ }^{*}$ Does not include sclerodactyly.

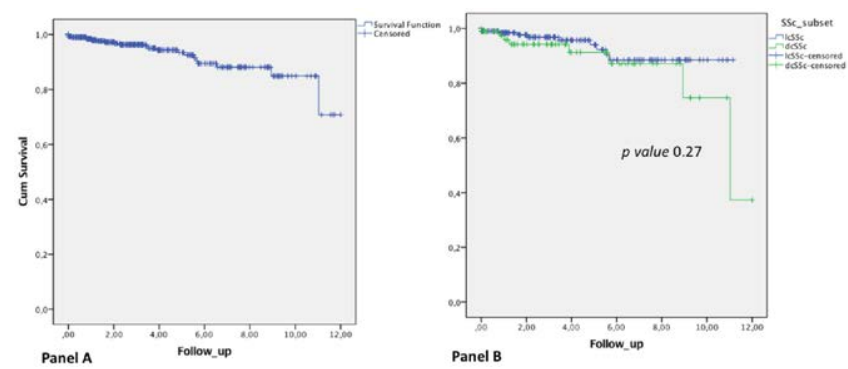

Figure 1. Panel A - Survival in years from diagnosis of patients with SSc included in Reuma $\mathrm{pt}$ in the first 2 years of disease ( $\mathrm{N}=472$ ). Panel B - survival according to SSc subset (IcSSc and dcSSC).

Disclosure of Interests: None declared DOI: 10.1136/annrheumdis-2021-eular.3159

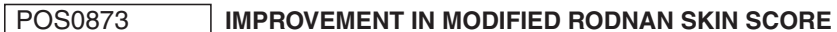 (MRSS) IN SYSTEMIC SCLEROSIS PATIENTS TREATED WITH RITUXIMAB: A SYSTEMATIC REVIEW AND META-ANALYSIS OF THE SCIENTIFIC LITERATURE}

J. L. Puga Guzmán ${ }^{1}$, D. Fernández Fernández ${ }^{2,3}$, R. Dos-Santos ${ }^{2,3}$, I. González Fernández ${ }^{2,3}$, E. Perez-Pampín ${ }^{3,4}$, A. Souto Vilas ${ }^{3,4}$, A. Mera Varela ${ }^{3,4}$ ${ }^{1}$ Facultade de Medicina e Odontoloxía - USC, Medicine, Santiago de Compostela, Spain; ${ }^{2}$ Hospital Clínico Universitario de Santiago, Rheumatology, Santiago de Compostela, Spain; ${ }^{3}$ University of Santiago de Compostela, Medicine, Santiago de Compostela, Spain; ${ }^{2}$ Hospital Clínico Universitario de Santiago, Rheumatology, Santiago de Compostela, Spain

Background: Systemic sclerosis (SSc) is a chronic autoimmune disease of the connective tissue characterized by vascular disease and fibrosis in different organs and systems such as lung and skin (1). Recently, several case reports and small series of patients reported on the efficacy of rituximab in SSc, showing a possible improvement in skin and lung affectations (2). However, registered clinical trials are lacking to determine factors associated with response, maintenance regimen, and long-term efficacy of rituximab in SSc.

Objectives: To analyze the efficacy of Rituximab in the treatment of skin fibrosis using the changes of the modified Rodnan Skin Score (mRSS) of patients diagnosed with systemic sclerosis from the data published in Registered Clinical Trials (RCTs) in the scientific literature.

Methods: We perform a systematic review and a meta-analysis using the main electronic databases to locate all the articles available so far: Medline, Embase, Cochrane Library and Web of science and ACR and EULAR abstracts congress were extracted to assess efficacy outcomes. That efficacy was measured based on the variation of mRSS at 12,24 and 48 weeks for patients treated with Rituximab versus patients treated with another drug or placebo. 